\title{
Análisis computacional del cocinado de carne a la plancha
}

\author{
Jara Moya, Begoña Calvo, Jorge Grasa \\ Applied Mechanics and Bioengineering - AMB- (T24-17R) \\ Instituto de Investigación en Ingeniería de Aragón (I3A) \\ Universidad de Zaragoza, Mariano Esquillor s/n, 50018, Zaragoza, Spain. \\ Tel.615551776, e-mail: jaram@unizar.es
}

\section{Resumen}

El análisis computacional de los procesos de cocinado puede suponer un ahorro de tiempo y dinero a la hora de recoger datos para la caracterización de los diferentes mecanismos implicados. La ventaja de estos modelos numéricos es el acceso a todas las variables involucradas en el cocinado en cualquier momento para comprender mejor la evolución de la transferencia de calor y de masa que tiene lugar en estos procesos. El objetivo de este estudio es desarrollar una metodología numérica que permita reproducir el comportamiento de la carne de tenera cuando se cocina a la plancha en una sarten. Para llevar a cabo esta simulación es necesario resolver un problema multifísico de transferencia de calor, transferencia de masa y mecánica de sólidos.

\section{Introducción}

El modelado de alimentos y el análisis de elementos finitos se han convertido en una herramienta importante para simular y comprender diferentes procesos de cocinado como hornear, asar (Llave et al., 2016), freír (Bansal et al. 2015) y cocinar en sartenes (Feyissa et al. 2011). Estos procesos de cocción están regidos básicamente por el acoplamiento entre los mecanismos de transferencia de calor y los mecanismos de transferencia de masa debido a la temperatura y el contenido de humedad. Cuando se calienta la comida, el agua migra a través de la superficie en forma de líquido o en forma de vapor, mientras que la temperatura y el contenido de agua dentro de la comida varían en el espacio y el tiempo, al mismo tiempo que su volumen. La distribución espacial final y también la evolución temporal influyen en la calidad y seguridad de los alimentos procesados. El objetivo de este estudio es definir y validar un modelo de elementos finitos que describa la transferencia de calor y transporte de agua durante el proceso de cocinado de carne de tenera a la plancha, incluyendo el proceso de darle la vuelta al filete.

\section{Modelo matemático}

El proceso de transferencia de calor dentro del producto, se puede modelar con un balance de energía único para todo el producto:

$$
\rho C_{p} \frac{\partial T}{\partial t}+\left(\vec{n}_{w, G} \cdot \nabla\left(C_{p, w} T\right)\right)=k \cdot(\lambda \nabla T)
$$

donde $\rho\left[\mathrm{kg} \mathrm{m}^{-3}\right]$ es la densidad del producto, $\mathrm{C}_{\mathrm{p}}$ y $\mathrm{C}_{\mathrm{p}}$, w son el calor específico del producto y el calor específico del agua respectivamente en $\left[\mathrm{Jkg}^{-1} \mathrm{~K}^{-1}\right]$, $\mathrm{n}_{\mathrm{w}, \mathrm{G}}$ es el flujo másico de agua respecto a un observador estacionario y $\mathrm{k}\left[\mathrm{Wm}^{-1} \mathrm{~K}^{-1}\right]$ es la conductividad térmica.

La carne se considera un material saturado, que no contiene fase gaseosa, donde el mecanismo principal es el transporte de agua. El frente de evaporación se limita a la superficie del material, por lo que no hay generación de vapor interna. Por lo tanto, las ecuaciones de conservación de masa se reducen solo a la del agua líquida:

$$
\frac{\partial c_{w}}{\partial t}+\nabla \cdot\left(c_{w} \vec{v}_{s, G}\right)=\nabla \cdot\left(D_{w} \nabla c_{w}+D_{w, T} \nabla T\right)
$$

donde $\mathrm{c}_{\mathrm{w}}\left[\mathrm{mol} \mathrm{m}^{-3}\right]$ es la concentración de agua, $\mathrm{v}_{\mathrm{s}, \mathrm{G}}$ es la velocidad de deformación del medio deformable en $\mathrm{m} / \mathrm{s}, \mathrm{D}_{\mathrm{w}}\left[\mathrm{m}^{2} \mathrm{~s}^{-1}\right]$ es la difusividad del agua y $D_{\mathrm{w}, \mathrm{T}}$ $\left[\mathrm{m}^{2} \mathrm{~s}^{-1}\right]$ es la difusividad del agua relacionada con la variación de temperatura.

Se ha utilizado un modelo de material hiperelástico para simular la retracción de la carne. La función de energía de deformación seleccionada ha sido la NeoHookean que, para materiales incompresibles, toma la forma:

$$
W_{e l}=\frac{K}{2}\left(J_{e l}-1\right)^{2}+\frac{\mu}{2}\left(\bar{I}_{1}-3\right)
$$

donde $\mathrm{K}[\mathrm{Pa}]$ y $\mu[\mathrm{Pa}]$ son la masa y el módulo elástico transversal, $\mathrm{J}_{\mathrm{el}}$ es el jacobiano elástico $\mathrm{y}_{1}$ es el primer invariante del tensor de Cauchy-Green derecho modificado. 


\section{Modelo de elementos finitos}

Se ha desarrollado un modelo 3D de elementos finitos mediante el software COMSOL Multiphysics 5.2. a para reproducir el proceso de cocinado. Este modelo incluye dos partes diferentes: una sartén de acero de $210 \mathrm{~mm}$ de diámetro y $5 \mathrm{~mm}$ de espesor y un filete de ternera, La muestra de carne se ha modelado como un cuboide rectangular en 3D de 19 $\mathrm{mm}$ de espesor. Para reproducir el proceso de darle la vuelta al filete se han considerado dos sartenes. Primero, la cara inferior del filete se calienta en una sartén. En segundo lugar, la cara superior del mismo trozo de carse se calienta en una segunda sartén. Se han simulado tres grados de cocinado de la carne: poco hecho (very rare), medio hecho (medium rare), y hecho (done), con diferentes tiempos de cocinado, y fijando el momento de darle la vuelta al filete en dos tercios del tiempo final de cocinado en cada caso.

\section{Resultados}

La Figura 1 muestra la evolución de la temperatura en el punto central del filete para el grado de cocinado medio hecho. Se observa que los resultados obtenidos mediante simulación se ajustan bien a los obtenidos experimentalmente, considerando un error por medición de la sonda de temperatura de $\Delta \delta=1$ $\mathrm{mm}$ respecto a la posición central. En cuanto a la pérdida de agua del filete, en la Figura 2 se observa que los resultados computacionales se ajustan nuevamente respecto a los experimentales. Posteriormente se ha comprobado que para espesores de $26 \mathrm{~mm}$ y de $34 \mathrm{~mm}$ el modelo de elementos finitos también reproduce bien la evolución de estas dos variables. De esta forma, se puede validar el modelo.

\section{Conclusiones}

Se ha desarrollado un modelo 3D de elementos finitos que considera los fenómenos de transferencia de calor y de agua y la deformación de la carne durante su cocinado a la plancha. Se han comparado la evolución en el tiempo de la temperatura en el punto central de la carne y la pérdida de peso predicha por el modelo con los resultados experimentales para diferentes grados de cocinado y espesores de carne. La buena concordancia entre los resultados previstos y los experimentales ha permitido verificar el modelo y considerar apropiados los supuestos formulados. Los resultados de las simulaciones proporcionan información detallada del cocinado a la plancha, lo que permitiría predecir con precisión el tiempo de cocinado requerido para alcanzar cierta temperatura en el centro de la carne, es decir, para lograr el grado de cocción deseado independientemente del grosor del filete de carne.

\section{REFERENCIAS}

[1]. Llave Y.; Takemori K.; Fukuoka M.; Takemori T.;Tomita H.; and Sakai N., 2016.Mathematical modeling of shrinkage deformation in eggplant undergoing simultaneous heat and mass transfer duringconvection-oven roasting.J Food Eng, 178, 124-136.

[2]. Bansal H.S.; Takhar P.S.; Alvarado C.Z.; andThompson L.D., 2015.Transport Mechanismsand Quality Changes During Frying of ChickenNuggetsHybrid Mixture Theory Based Modelingand Experimental Verification.J Food Sci, 80,no. 12, E2759-E2773.

[3]. Feyissa A.H.; Gernaey K.V.; Ashokkumar S.; andAdler-Nissen J., 2011.Modelling of coupled heatand mass transfer during a contact baking process.Journal of Food Engineering, 106, no. 3, 228235.

\section{FIGURAS / LEYENDAS}

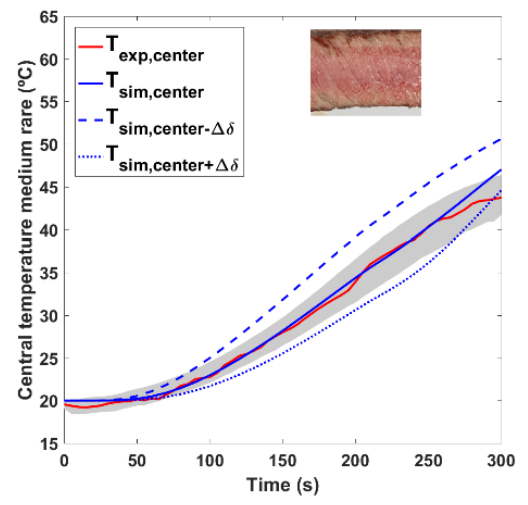

Figura 1. Evolución de la temperatura en el punto central de la carne para el caso de cocinado medio hecho.

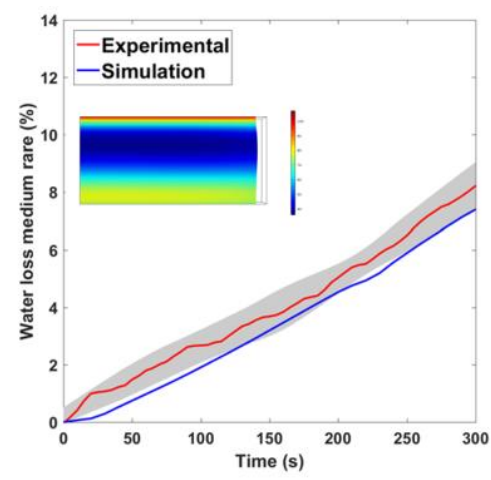

Figura 2. Evolución de la pérdida de agua de la carne para el caso de cocinado medio hecho. 